\title{
Bonding of a Carbon Nanotube Film to a Au Film at Low Temperature and Contact Resistance of the Film under Micronewton Loads
}

\author{
Hiroshi Kinoshita ${ }^{*}$, Yoshitada Isono and Nobuo Ohmae \\ Department of Mechanical Engineering, Faculty of Engineering, Kobe University \\ 1-1 Rokkodai, Nada-Ku, Kobe 657-8501, Japan \\ *Corresponding author: kinohiro@people.kobe-u.ac.jp
}

( Manuscript received 6 Junuary 2011; accepted 3 February 2011; published 15 February 2011 )

\begin{abstract}
A process for bonding a carbon nanotube (CNT) film to a Au film at low temperature was developed with a view to using the CNT films as electrical contact materials in radio-frequency microelectromechanical systems (RF MEMS) switches. First, a CNT film was synthesized on a silicon substrate at $750{ }^{\circ} \mathrm{C}$ and subsequently coated with $\mathrm{Au}$ at room temperature. Finally, the Au-coated CNT film was bonded at $100{ }^{\circ} \mathrm{C}$ to a $\mathrm{Au}$ film, which was then deposited on a silicon substrate to act as a transmission line. An apparatus was developed to measure contact resistances and forces under contact conditions mimicking those that will be encountered in actual RF MEMS switches, such as micronewton loads and square-micrometer contact areas. Contact resistance and force of the bonded CNT film sample were simultaneously measured as a function of displacement between the sample and an electrode Au tip and compared with those for a Au film and $\mathrm{Au}$-coated CNT film. The bonded CNT film sample had no adhesion force, but higher contact resistance than the other samples.
\end{abstract}

Keywords: RF MEMS, carbon nanotube, contact resistance, contact load

\section{Introduction}

As compared to solid-state semiconductor switches, radio-frequency microelectromechanical systems (RF MEMS) switches have potentially superior electrical properties such as low insertion loss, good isolation, low power consumption, and good linearity, especially in the high frequency range ${ }^{1,2)}$. Although RF MEMS switches possess these advantages, there are problems with the electrical contacts: The mechanical relay switches in RF MEMS switches are microsized, and so the contact electrodes must be considerably smaller (less than 100 $\mu \mathrm{m}^{2}$ ) than those of conventional relay switches. In addition, they can bear only low loads ranging from a few micronewton to several millinewton. Therefore, it is difficult to ensure reliable electrical contacts for RF MEMS switches, particularly for hundreds of billions of contact cycles. In order to address these problems, tribological knowledge and technology are necessary.

The materials used in the contact electrodes play an important role in solving the potential problems of RF MEMS switches. Although $\mathrm{Au}$ is one of the candidate materials for the contact electrodes ${ }^{3)}$, it has a high adhesion force and is easily deformed under relatively low loads. Carbon nanotubes (CNT) have not only excellent mechanical properties ${ }^{4,5)}$ but also excellent electrical properties, which include high current density $\left(>10^{9} \mathrm{~A} / \mathrm{cm}^{2}\right)$ and high resistance stability at $250{ }^{\circ} \mathrm{C}^{6)}$. Fundamental experiments for measurement of contact resistances of CNT films ${ }^{7-9)}$ and application of CNT films as contact electrodes for RF MEMS switches ${ }^{9)}$ have been carried out. However, these experiments used CNT films that were synthesized directly on substrates at high temperature $\left(>700{ }^{\circ} \mathrm{C}\right)$. These high-temperature processes are not suitable for MEMS processes. In order to apply CNT films to contact electrodes of RF MEMS switches, low temperature process to build CNT films on RF MEMS switches is absolutely necessary. In addition, these experiments used relatively high contact loads $(>1 \mathrm{mN})$, which caused plastic deformation of the CNTs.

In order to develop the low temperature process, we attempted to bond CNT film on an Au film used as transmission line at low temperature. Contact resistances and forces of the bonded CNT film, an Au film, and an $\mathrm{Au}$ coated $\mathrm{CNT}$ film under $\mu \mathrm{N}$ loads were simultaneously measured as a function of displacement between the samples and an electrode $\mathrm{Au}$ tip by a contact resistance measurement apparatus developed in this study. 


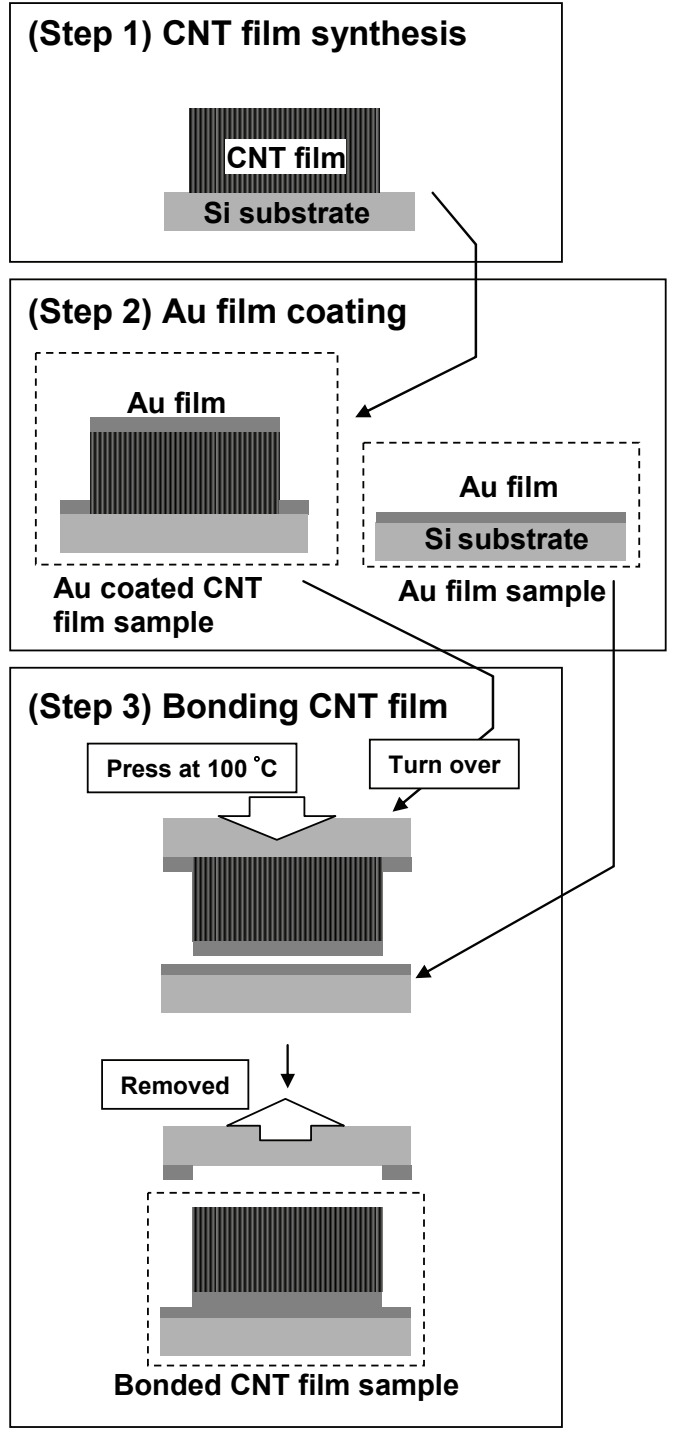

Fig. 1 Schematic of the three steps for the formation of the bonded CNT film sample

\section{Sample preparation}

The bonding of a CNT film to an $\mathrm{Au}$ film was carried out by 3 steps as schematically shown in Fig. 1 .

Step 1 (Synthesis of CNT film): In this study, we used silicon substrates with silicon oxide films of 100 $\mathrm{nm}$ thickness, which were formed by thermal oxidation. Deposition and annealing of an iron layer of $4 \mathrm{~nm}$ thickness were conducted on the silicon substrate to form catalytic nanoparticles. A mixture of $\mathrm{C}_{2} \mathrm{H}_{2}$ and $\mathrm{H}_{2}$ gases was flowed at the substrate temperature of $750{ }^{\circ} \mathrm{C}$ for $20 \mathrm{~min}$ at a pressure of $300 \mathrm{~Pa}$. Thus, CNT films consisting of multi-walled CNTs were synthesized on the silicon substrate. Figs. 2 (a) and (b) show the higher and lower magnification SEM images of the as-synthesized CNT film on the silicon substrate. The thickness of the CNT film was approximately $110 \mu \mathrm{m}$. As shown in Figs. 2 (a) and (b), CNTs were seen to be aligned vertically at low magnification, but at higher
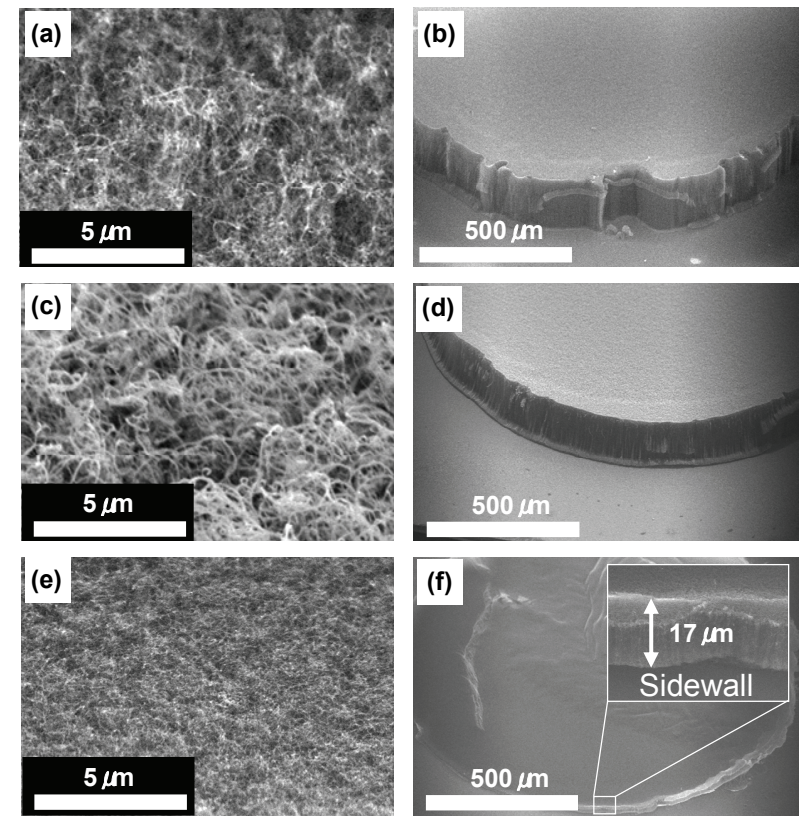

Fig. 2 SEM images of CNT films. (a,b) Synthesized CNT film on the silicon substrate. $(c, d)$ Au-coated CNT film. (e,f) Bonded CNT film.

magnification, a tangled structure was clearly seen.

Step 2 (Coating with Au film): A transmission line was synthesized by forming a $\mathrm{Au}$ film of approximately $30 \mathrm{~nm}$ thickness on the silicon substrate using a vacuum evaporator. We attempted simply to bond the as-synthesized CNT film to the $\mathrm{Au}$ film; however, the result was not successful. Au was therefore also coated on the as-synthesized CNT film in order to make it easy to bond the CNT film to bond to the $\mathrm{Au}$ film in the subsequent step. Figs. 2 (c) and (d) show the higher and lower magnification SEM images of the Au-coated CNT film sample.

Step 3 (Bonding of the CNT films to the Au film): The Au-coated CNT film was turned over and bonded to the $\mathrm{Au}$ film (as shown in Fig. 1), i.e., the Au-coating face of the Au-coated CNT film and the Au film were bonded with loads on the order of a few newton at a temperature of $100{ }^{\circ} \mathrm{C}$. The substrate on which the CNT film was synthesized was then removed at room temperature. Finally, a CNT film bonded to an Au film was obtained. Figs. 2 (e) and (f) show the higher and lower magnification SEM images of the bonded CNT film sample. The inset of Fig. 2 (f) shows the SEM image of the sidewall of the bonded CNT film. It appears that the CNTs were still aligned vertically after the pressing process. The thickness was decreased to be approximately $17 \mu \mathrm{m}$ by the load. The top face of the bonded CNT film was originally the bottom of the as-synthesized CNT film, and appears to be more flat and of a higher density than that of the as-synthesized CNT film.

In these steps, we formed three samples as enclosed by dashed line boxes in Fig. 1: the $\mathrm{Au}$ film, the $\mathrm{Au}-$ coated CNT film, and the bonded CNT film samples. 


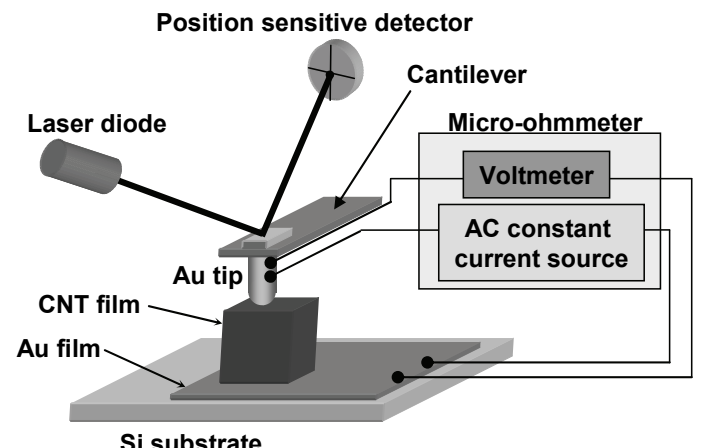

Fig. 3 Schematic drawing of the contact resistance measurement apparatus

\section{Contact resistance measurement method}

Figure 3 shows a schematic drawing of the contact resistance measurement apparatus. To measure low force $(<1 \mu \mathrm{N})$, the apparatus has a phosphor-bronze cantilever that is $1 \mathrm{~mm}$ wide, $6 \mathrm{~mm}$ long, and $0.05 \mathrm{~mm}$ thick. Additionally, the apparatus has a laser diode and a position sensitive detector for the optical lever method ${ }^{10)}$. A Au tip with an apex radius of $25 \mu \mathrm{m}$ was used as a contact electrode to realize a small contact area. The tip was formed by electrochemical etching, then cleaned in ethanol for $5 \mathrm{~min}$ and in water for $5 \mathrm{~min}$. Displacement between the samples and the Au tip was adjusted by a PZT actuator with closed-loop control. Contact resistances (Rc) were measured by the four-wire measurement method. An AC constant current source provided a steady output current on the substrates of 1.5 $\mu \mathrm{A}$ at $1 \mathrm{kHz}$ between the Au tip and the Au transmission line, independent of resistances. Measurements were carried out in air at a temperature of $25^{\circ} \mathrm{C}$ and a relative humidity of $25 \%$.

\section{Contact resistances and forces}

Figure 4 represents the contact resistances and forces of the three samples as a function of displacement between the sample and the $\mathrm{Au}$ tip. Dashed and solid lines show contact resistances and loads, respectively. The approaching and the retracting curves are indicated by black and grey, respectively. The maximum load was $11.5 \mu \mathrm{N}$. The zero of displacement was defined at the value where the load reached the maximum value.

For the Au film sample shown in Fig. 4 (a), the lowest contact resistance was $2 \Omega$. An adhesion force of 6.5 $\mu \mathrm{N}$ was observed, which arose from water adsorption between the Au tip and the film. As shown in Fig. 4 (b), the lowest contact resistance of the bonded CNT film sample was $636 \Omega$, which was much higher than that of the Au film sample. There was no adhesion force for the bonded CNT film sample, which may be the result of less water adsorption ${ }^{11)}$. The lowest contact resistance of the Au-coated CNT film sample in Fig. 4 (c) was $72 \Omega$, which was lower than that of the CNT film, but higher than that of the Au film sample. The

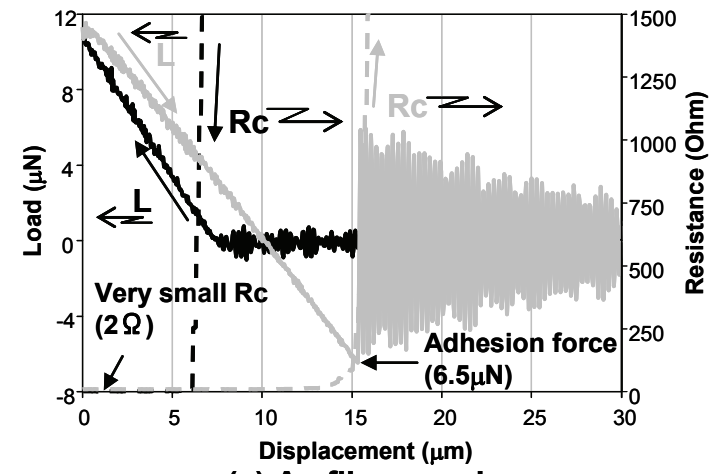

(a) Au film sample

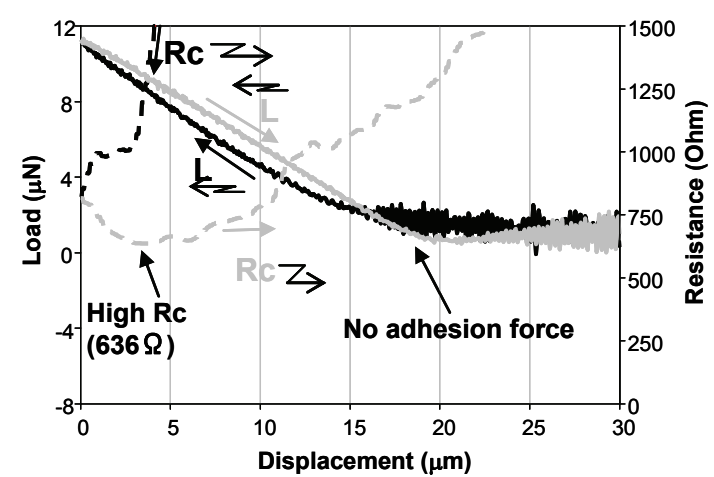

(b) Bonded CNT film sample

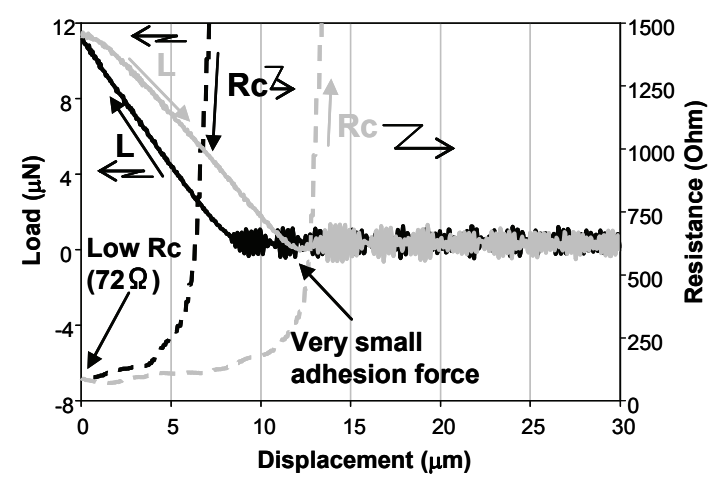

(c) Au coated CNT film sample

Fig. 4 Relationships between contact resistances and loads. (a) Au film sample. (b) Bonded CNT film sample. (c) Au coated CNT film sample.

adhesion force was observed but it was very small ( $<$ $0.1 \mu \mathrm{N})$. As shown in Fig. 4, hystereses of the contact resistances and forces of all these samples were observed. These hystereses did not arise from the PZT actuator, as a closed-loop PZT actuator was used. The cause of the hysteresis is unfortunately unknown to us at this time.

The contact resistance of the bonded CNT film sample was high and should be decreased for RF MEMS switches. Yunus et al. previously reported that contact resistances of the sputter-coated $\mathrm{Au}$ film, as-grown CNT film, and Au-coated CNT film were $0.39 \Omega, \sim 108 \Omega, \sim 0.46 \Omega$, respectively, with the $\phi 2 \mathrm{~mm}$ 
Au-sputter-coated stainless ball at the maximum load of $1 \mathrm{mN}^{9)}$. These contact resistances were lower than those in our study, which may result from the larger ball and higher load, i.e., a larger contact area in comparison with our study. The resistances of individual CNTs with $\mathrm{Au} / \mathrm{Ti}$ electrodes were dominated by the contact resistance and were not significantly affected by the intrinsic resistance of the $\mathrm{CNTs}^{12}$. It was also reported that contact resistances of the individual CNTs that were not tightly deposited on $\mathrm{Au}$ were over $100 \mathrm{M} \Omega^{13)}$. Contact resistances of individual CNTs on which tungsten electrodes were tightly deposited were on the order of a few kilo-ohm ${ }^{6}$. These results indicate that when CNTs that have not been tightly deposited onto the substrate make contact with electrode surfaces, their contact resistances are high. In the bonded CNT film sample, individual CNTs were in contact only with the electrode Au tip, thus, the contact resistance was high. For the Au-coated CNT film sample in this study, the $\mathrm{Au}$ tip was in contact with the Au coating that was tightly deposited on the individual CNTs. The Au coating provided lower contact resistance. Contact resistances between CNTs and metals depend on metal elements. For example, bondings of $\mathrm{Sc}, \mathrm{Ti}$, and $\mathrm{V}$ on single-walled CNTs are expected to have low contact resistances ${ }^{14)}$. In order to decrease the contact resistance of the bonded CNT film sample, experiments for various metal coatings should be carried out.

Since the reliability of RF MEMS switches is very important for their practical applications, future experiments for long-term operation will be carried out.

\section{Summary}

In this study, a process was developed for bonding a CNT film to a Au film at $100{ }^{\circ} \mathrm{C}$ such that it is suitable for MEMS devices. A contact resistance measurement apparatus for micronewton loads and square-micrometer areas was also developed. Contact resistances and forces of the bonded CNT film sample, an Au film sample, and an Au-coated CNT film sample were measured and compared. The bonded CNT film sample had no adhesion force, but exhibited higher contact resistance than the other samples.

\section{Acknowledgment}

This study is financially supported by Grant-in-Aid for Scientific Research (C) (KAKENHI (22560142)).

\section{References}

[1] Yao, Z., Chen, S., Eshelman, S., Denniston, D. and Goldsmith, C., "Micromachined Low Loss Microwave Switches," IEEE Journal of Microelectromechanical Systems, 8, 2, 1999, 129-134.

[2] Park, J. H., Lee, H. C., Park, Y. H., Kim, Y. D., Ji, C. H., Bu, J. and Nam, H. J., “A Fully Wafer-Level
Packaged RF MEMS Switch with Low Actuation Voltage Using a Piezoelectric Actuator," Journal of Micromechanics and Microengineering, 16, 2006, 2281-2286.

[3] Patton, S. T. and Zabinski, J. S., "Fundamental Studies of Au Contacts in MEMS RF Switches," Tribology Letters, 18, 2, 2005, 215-230.

[4] Yu, M. F., Lourie, O., Dyer, M. J., Moloni, K., Kelly, T. F. and Ruoff, R. S., "Strength and Breaking Mechanism of Multiwalled Carbon Nanotubes under Tensile Load," Science, 287, 2000, 637-640.

[5] Wong, E. W., Sheehan, P. E. and Lieber, C. M., "Nanobeam Mechanics: Elasticity, Strength, and Toughness of Nanorods and Nanotubes," Science, 277, 1997, 1971-1975.

[6] Wei, B. Q., Vajtai, R. and Ajayan, P. M., "Reliability and Current Carrying Capacity of Carbon Nanotubes," Applied Physics Letters, 79, 8, 2001, 1172-1174.

[7] Tzeng, Y., Chen, Y. and Liu, C., "Electrical Contacts between Carbon-Nanotube Coated Electrodes," Diamond and Related Materials, 12, 2003, 774-779.

[8] Yaglioglu, O., Hart, A. J., Martens, R. and Slocum, A. H., "Method of Characterizing Electrical Contact Properties of Carbon," Review of Scientific Instruments, 77, 2006, 095105.

[9] Yunus, E. M., McBride, J. W. and Spearing, S. M., "The Relationship between Contact Resistance and Contact Force on Au-Coated Carbon Nanotube Surface under Low Force Condition," IEEE Transactions on Components and Packaging Technologies, 32, 3, 2009, 650-657.

[10] Baba, Y., Kinoshita, H., Tagawa, M., Umeno, M. and Ohmae, N., "Water Adsorption on the LIGA Ni Film and Its Effects on Friction," Tribologist, 44, 5, 1999, 379-386. (in Japanese)

[11] Turqa, V., Ohmae, N., Martin, J. M., Fontaine, J., Kinoshita, H. and Loubet, J. L., "Influence of Humidity on Microtribology of Vertically Aligned Carbon Nanotube Film," Tribology Letters, 19, 1, 2005, 23-28.

[12] Wakaya, F., Katayama, K. and Gamo, K., "Contact Resistance of Multiwall Carbon Nanotubes," Microelectronic Engineering, 67-68, 2003, 853-857.

[13] Bachtold, A., Henny, M., Terrier, C., Strunk, C., Schönenberger, C., Salvetat, J. P., Monard, J. M. and Forró, L., "Contacting Carbon Nanotubes Selectively with Low-Ohmic Contacts for Four-Probe Electric Measurements," Applied Physics Letters, 73, 2, 1998, 274-27.

[14] Andriotis, A. N., Menon, M. and Froudakis, G. E., "Various Bonding Configurations of Transition-Metal Atoms on Carbon Nanotubes: Their Effect on Contact Resistance," Applied Physics Letters, 76, 26, 2000, 3890-3892. 